\title{
Some Aspects of Geometric Constants in Modular Spaces
}

\author{
Zhijian Yang, Qi Liu, Muhammad Sarfraz, Yongjin Li* \\ Department of Mathematics, Sun Yat-sen University, Guangzhou, 510275, P. R. China \\ yangzhj55@mail2.sysu.edu.cn, liuq325@mail2.sysu.edu.cn, sarfraz@mail2.sysu.edu.cn, \\ stslyj@mail.sysu.edu.cn \\ *Correspondence: stslyj@mail.sysu.edu.cn
}

\begin{abstract}
In this paper, we generalize the typical geometric constants of Banach spaces to modular spaces. We study the equivalence between the convexity of modular and normed spaces, and obtain the relationship between $\rho$-Neumann-Jordan constant and $\rho$-James constant. In particular, we extend the convexity and smoothness modular, and obtain the criterion theorems of the uniform convexity and strict convexity.
\end{abstract}

\section{INTRODUCTION}

In the recent years, the geometric theory of Banach spaces has been fully developed, especially the geometric constant, which is a powerful tool to characterize the geometric properties of the space sphere. As early as 1936, Clarkson introduced the convexity modular of space [1]. In 1963, Lindenstrauss introduced the smoothness modular, and obtained the close relationship between the two constants [2]. In 1937, in order to better characterize Jordan and von-Nuemann's famous work in inner product spaces, Clarkson defines the von-Nuemann constant [3] which is the minimum constant $C$ for all $x, y \in X$ and $(x, y) \neq(0,0)$ of the following equations:

$$
\frac{1}{C} \leq \frac{\|x+y\|^{2}+\|x-y\|^{2}}{2\left(\|x\|^{2}+\|y\|^{2}\right)} \leq C .
$$

In 1964, James introduced James constant [4] in order to study the normal structure of space. After the appearance of these constants, many scholars paid attention to them and obtained many wonderful properties [5].

Modular space problems have been considered by H. Nakano, Musielak and Orlicz [6] under the additional hypothesis of convexity or subadditivity of the modular $\rho: X \rightarrow[0,+\infty)$. Moreover the case of semi-ordered linear spaces and that of B-norms have been chiefly investigated. Under

Received: 13 Sep 2021.

Key words and phrases. Banach spaces; geometric constants; modular spaces. 
weaker assumptions, they investigated the structure of the spaces under consideration. Neither convexity nor subadditivity of the modular be assumed. In introducing the norm, a certain natural connection between the modular and the norm convergence will be required: norm convergence should imply modular convergence.

Through their researches, they found that although modular spaces are not generally normed spaces, they still have many wonderful properties, such as convergence, completeness, convexity and additivity. In view of these properties, Poom Kumam extended Jordan Von-Neumann constant and James constant in Banach spaces to modular spaces, and obtained uniform convexity and uniform non-squareness of modular spaces [10].

In this paper, based on the idea of generalizing geometric constants in Banach spaces to modular spaces, we generalize the properties of von-Neumann constant and James constant in [10]. By defining convexity modules and smoothness modular, we derive the relationships between James constant, convexity modular and the strict convexity of modular spaces.

\section{Preliminaries}

We first give some basic facts about modular spaces formulated by Musielak and Orlicz [6]. Definition 1.[8] Let $X$ be a vector space over $F(R$ or $C)$. Then a function $\rho: X \rightarrow[0, \infty]$ is called a modular on $X$ if for arbitrary $x, y$ in $X$,

(i) $\rho(x)=0$ if and only if $x=0$,

(ii) $\rho(\alpha x)=\rho(x)$ for every scalar $\alpha$ with $|\alpha|=1$,

(iii) $\rho(\alpha x+\beta y) \leq \rho(x)+\rho(y)$ if $\alpha+\beta=1$ and $\alpha, \beta \geq 0$.

If (iii) is replaced by (iv): $\rho(\alpha x+\beta y) \leq \alpha \rho(x)+\beta \rho(y)$ if $\alpha, \beta \geq 0$ and $\alpha+\beta=1$. We now call that $\rho$ is a convex modular.

A modular $\rho$ can be used to define a corresponding modular space, i.e, the vector space $X_{\rho}$ as given by

$$
X_{\rho}=\{x \in X: \rho(\lambda x) \rightarrow 0 \text { as } \lambda \rightarrow 0\}
$$

where $X_{\rho}$ is a linear subspace of $X$.

In general, the modular $\rho$ is not necessarily subadditive and therefore it does not behave as a norm or a distance. But we can associate it to a modular $F$-norm.

The modular space $X_{\rho}$ can be equipped with a $F$-norm defined by

$$
\|x\|_{\rho}=\inf \left\{\alpha>0 ; \rho\left(\frac{x}{\lambda}\right) \leq \alpha\right\}
$$

when $\rho$ is convex. Then norm $\|\cdot\|_{\rho}$ is frequently called the Luxemburg norm. If $\rho$ is convex, then the functional $\|x\|_{\rho}=\inf \left\{\alpha>0 ; \rho\left(\frac{x}{\lambda}\right) \leq 1\right\}$ is a norm in $X_{\rho}$ which is equivalent to the $F$-norm $\|\cdot\|_{\rho}$ 
Proposition 1. Let $X_{\rho}$ be a modular space. Then $\rho$ is convex if and only if $X_{\rho}$ is a normed space with $\rho$ as norm.

Proof. The proof of sufficiency is obvious.

Conversely, assume $\rho$ is convex, then we can obtain $\rho(x)=0$ if and only if $x=0$.

(i) According to the Definition 1, if $\alpha>0$, then

$$
\rho\left(\frac{1}{\alpha} x\right)=\rho\left(\frac{1}{\alpha} x+\frac{1-\alpha}{\alpha} \cdot 0\right) \leq \frac{1}{\alpha} \rho(x)+\frac{1-\alpha}{\alpha} \rho(0)=\frac{1}{\alpha} \rho(x)
$$

and

$$
\alpha \rho\left(\frac{1}{\alpha} x\right)=\alpha \rho\left(\frac{1}{\alpha} x\right)+(1-\alpha) \rho(0) \geq \rho\left(\alpha \cdot \frac{1}{\alpha}+(1-\alpha) \cdot 0\right)=\rho(x) .
$$

This show that $\rho\left(\frac{1}{\alpha} x\right) \geq \frac{1}{\alpha} \rho(x)$ and hence $\rho\left(\frac{1}{\alpha} x\right)=\frac{1}{\alpha} \rho(x)$ for $\alpha>0$.

Suppose $\alpha \neq 0$, then $|\alpha|>0$. According to the Definition 1 , we have

$$
\rho\left(|\alpha| \cdot \frac{1}{|\alpha|} \alpha x\right)=|\alpha| \rho\left(\frac{1}{|\alpha|} \alpha x\right)=|\alpha| \rho(x)
$$

which shows that $\rho(\alpha x)=|\alpha| \rho\left(\frac{1}{|\alpha|} \alpha x\right)=|\alpha| \rho(x)$.

(ii) Since

$$
\rho(x+y)=\rho\left(2\left(\frac{x}{2}+\frac{y}{2}\right)\right)=2 \rho\left(\frac{x}{2}+\frac{y}{2}\right) \leq \rho(x)+\rho(y),
$$

then $X_{\rho}$ is a normed space with $\rho$ as norm.

\section{The $\rho$-Neumann-Jordan constant AND the $\rho$-James constant}

In 2006, Poom Kumam [10] generalized two typical constants

$$
C_{N J}(X)=\sup \left\{\frac{\|x+y\|^{2}+\|x-y\|^{2}}{2\|x\|^{2}+2\|y\|^{2}}: x, y \in X,(x, y) \neq(0,0)\right\}
$$

and

$$
J(X)=\sup \{\min \{\|x+y\|,\|x-y\|\}: x, y \in X,\|x\|=\|y\|=1\}
$$

and introduced two new geometric constants $C_{N J}\left(X_{\rho}\right)$ and $J\left(X_{\rho}\right)$ defined on modular spaces.

Definition 2.[10] The $\rho$-Neumann-Jordan constant $C_{N J}\left(X_{\rho}\right)$ of a modular space $X_{\rho}$ is defined by

$$
C_{N J}\left(X_{\rho}\right)=2 \sup \left\{\frac{\rho^{2}\left(\frac{x+y}{2}\right)+\rho^{2}\left(\frac{x-y}{2}\right)}{\rho^{2}(x)+\rho^{2}(y)}: x, y \in X_{\rho}, \rho(x)=1, \rho(y) \leq 1\right\} \text {. }
$$

Definition 3.[10] The $\rho$-James constant $J\left(X_{\rho}\right)$ of a modular space $X_{\rho}$ is defined by

$$
J\left(X_{\rho}\right)=2 \sup \left\{\min \left\{\rho\left(\frac{x+y}{2}\right), \rho\left(\frac{x-y}{2}\right)\right\}: x, y \in X_{\rho}, \rho(x)=1, \rho(y) \leq 1\right\} .
$$

In the following section, we extend the Proposition 3.5 in [10] and obtain inequalities of $C_{N J}\left(X_{\rho}\right)$ and $J\left(X_{\rho}\right)$.

Theorem 1. Let $X_{\rho}$ be a modular space, then 
(i) $0<J\left(X_{\rho}\right) \leq 4$ and $1 \leq C_{N J}\left(X_{\rho}\right) \leq 8$, in particular, if $\rho$ is convex, then $1 \leq J\left(X_{\rho}\right) \leq 2$ and $1 \leq C_{N J}\left(X_{\rho}\right) \leq 2$

(ii) $\frac{1}{2} J^{2}\left(X_{\rho}\right) \leq C_{N J}\left(X_{\rho}\right) \leq \frac{64}{J^{2}\left(X_{\rho}\right)}+4$, in particular, if $\rho$ is convex, then $\frac{1}{2} J^{2}\left(X_{\rho}\right) \leq C_{N J}\left(X_{\rho}\right) \leq$ $\frac{4}{J^{2}\left(X_{\rho}\right)}+1$.

Proof. (i) Let $y=0$, then

$$
J\left(X_{\rho}\right) \geq 2 \sup \left\{\rho\left(\frac{x}{2}\right): x \in X_{\rho}, \rho(x)=1\right\} .
$$

Since $\rho(x)=1$, then $\rho\left(\frac{x}{2}\right)>0$ implies $J\left(X_{\rho}\right)>0$. Since $\rho\left(\frac{x \pm y}{2}\right) \leq \rho(x)+\rho(y) \leq 2$, then $0<J\left(X_{\rho}\right) \leq 4$.

Let $x=y$, then

$$
C_{N J}\left(X_{\rho}\right) \geq 2 \sup \left\{\frac{\rho^{2}\left(\frac{x+x}{2}\right)+\rho^{2}\left(\frac{x-x}{2}\right)}{\rho^{2}(x)+\rho^{2}(x)}: x \in X_{\rho}, \rho(x)=1\right\} \geq 1 .
$$

Since $\rho^{2}\left(\frac{x+y}{2}\right)+\rho^{2}\left(\frac{x-y}{2}\right) \leq 2(1+\rho(y))^{2}$, we have

$$
\frac{\rho^{2}\left(\frac{x+y}{2}\right)+\rho^{2}\left(\frac{x-y}{2}\right)}{\rho^{2}(x)+\rho^{2}(y)} \leq 2\left(1+\frac{2 \rho(y)}{1+\rho^{2}(y)}\right) \leq 4,
$$

thus $1 \leq C_{N J}\left(X_{\rho}\right) \leq 8$.

In particular, if $\rho$ is convex and let $x=y$, then

$$
J\left(X_{\rho}\right) \geq 2 \sup \left\{\rho\left(\frac{x}{2}\right): x \in X_{\rho}, \rho(x)=1\right\}=1 .
$$

Since $\rho\left(\frac{x \pm y}{2}\right) \leq \frac{1}{2} \rho(x)+\frac{1}{2} \rho(y) \leq 1$, then $1 \leq J\left(X_{\rho}\right) \leq 2$. We also can prove $1 \leq C_{N J}\left(X_{\rho}\right) \leq 2$ by the same way.

(ii) Since

$$
\rho^{2}\left(\frac{x+y}{2}\right)+\rho^{2}\left(\frac{x-y}{2}\right) \leq 2[1+\rho(y)]^{2} \leq 4\left(1+\rho^{2}(y)\right)
$$

then

$$
\frac{\rho^{2}\left(\frac{x+y}{2}\right)+\rho^{2}\left(\frac{x-y}{2}\right)}{\rho^{2}(x)+\rho^{2}(y)}-2 \leq \frac{2(1+\rho(y))^{2}}{1+\rho^{2}(y)}-2=\frac{4 \rho(y)}{1+\rho^{2}(y)} .
$$

Since $\frac{1}{4}\left(\rho^{2}\left(\frac{x+y}{2}\right)+\rho^{2}\left(\frac{x-y}{2}\right)\right) \leq 1+\rho^{2}(y)$, then $\frac{4 \rho(y)}{1+\rho^{2}(y)} \leq \frac{16 \rho(y)}{\rho^{2}\left(\frac{x+y}{2}\right)+\rho^{2}\left(\frac{x-y}{2}\right)}$, that is

$$
\begin{aligned}
& \frac{\rho^{2}\left(\frac{x+y}{2}\right)+\rho^{2}\left(\frac{x-y}{2}\right)}{\rho^{2}(x)+\rho^{2}(y)}-2 \\
& \leq \frac{16 \rho(y)}{\rho^{2}\left(\frac{x+y}{2}\right)+\rho^{2}\left(\frac{x-y}{2}\right)} \\
& \leq \frac{16}{\rho^{2}\left(\frac{x+y}{2}\right)+\rho^{2}\left(\frac{x-y}{2}\right)} .
\end{aligned}
$$

Finally $\frac{1}{2} C_{N J}\left(X_{\rho}\right)-2 \leq \frac{16}{\frac{1}{2} J^{2}\left(X_{\rho}\right)}$ implies that $C_{N J}\left(X_{\rho}\right) \leq \frac{64}{J^{2}\left(X_{\rho}\right)}+4$. 
According to the proof of Proposition 3.5 in [12], we can prove $\frac{1}{2} J^{2}\left(X_{\rho}\right) \leq C_{N J}\left(X_{\rho}\right)$, thus

$$
\frac{1}{2} J^{2}\left(X_{\rho}\right) \leq C_{N J}\left(X_{\rho}\right) \leq \frac{64}{J^{2}\left(X_{\rho}\right)}+4
$$

In particular, if $\rho$ is convex, then

$$
\rho^{2}\left(\frac{x+y}{2}\right)+\rho^{2}\left(\frac{x-y}{2}\right) \leq \frac{1}{2}(1+\rho(y))^{2} \leq 1+\rho^{2}(y),
$$

thus

Therefore

$$
\frac{\rho^{2}\left(\frac{x+y}{2}\right)+\rho^{2}\left(\frac{x-y}{2}\right)}{\rho^{2}(x)+\rho^{2}(y)}-\frac{1}{2} \leq \frac{\rho(y)}{1+\rho^{2}(y)} \leq \frac{1}{\rho^{2}\left(\frac{x+y}{2}\right)+\rho^{2}\left(\frac{x-y}{2}\right)} .
$$

$$
C_{N J}\left(X_{\rho}\right) \leq \frac{4}{J^{2}\left(X_{\rho}\right)}+1
$$

Example 1. (i) Consider $X=R^{2}, \rho(x)=\left\{\begin{array}{c}0, x=0 \\ \frac{1}{\|x\|_{1}}, x \neq 0\end{array}\right.$, where $\|x\|_{1}=\left\|\left(x_{1}, x_{2}\right)\right\|_{1}=\left|x_{1}\right|+\left|x_{2}\right|$. Obviously, $X_{\rho}$ is a modular space.

We choose $x_{0}=\left(\frac{1}{2}, \frac{1}{2}\right), y_{0}=\left(\frac{1}{2},-\frac{1}{2}\right)$, then

$$
\rho\left(x_{0}\right)=\rho\left(y_{0}\right)=1, \rho\left(\frac{x_{0}+y_{0}}{2}\right)=\rho\left(\frac{x_{0}-y_{0}}{2}\right)=2,
$$

thus $J\left(X_{\rho}\right) \geq 4$. Since $J\left(X_{\rho}\right) \leq 4$, then $J\left(X_{\rho}\right)=4$. According to (ii) of Theorem 1 , we know that $C_{N J}\left(X_{\rho}\right)=8$ in this example.

(ii) Consider $X=R^{2}, \rho(x)=\|x\|_{1}$. Obviously, $X_{\rho}$ is a modular space and $\rho$ is convex. We have

$$
J\left(X_{\rho}\right)=\sup \left\{\min \left\{\|x+y\|_{1},\|x-y\|_{1}\right\}: x, y \in X_{\rho},\|x\|=1,\|y\| \leq 1\right\} .
$$

We choose $x_{0}=(1,0), y_{0}=(0,1)$, then $\left\|x_{0}\right\|_{1}=\left\|y_{0}\right\|_{1}=1$ and $\left\|x_{0}+y_{0}\right\|_{1}=\left\|x_{0}-y_{0}\right\|_{1}=2$, thus $J\left(X_{\rho}\right)=2$. According to (ii) of Theorem 1, we can get that $C_{N J}\left(X_{\rho}\right)=2$ in this example.

\section{THE $\rho$-CONVEX MODULAR AND THE $\rho$-SMOOTH MOdULAR}

In order to study the uniform convexity of Banach spaces, Clarkson introduced the modular of convexity

$$
\delta_{X}(\varepsilon)=\inf \left\{1-\frac{1}{2}\|x+y\|:\|x\|=\|y\|=1,\|x-y\| \geq \varepsilon\right\} .
$$

Goebel called $\varepsilon_{0}=\sup \left\{\varepsilon \in[0,2]: \delta_{X}(\varepsilon)=0\right\}$ as the characteristic of convexity. Based on the geometric intuitionistic meaning of convexity of Banach spaces and its application in fixed point theory, this paper gives the $\rho$-convex modular of modular spaces with reference to the definition of $\delta_{X}(\varepsilon)$.

Definition 4. The $\rho$-convex modular $\delta_{X_{\rho}}(\varepsilon)$ of a modular space $X_{\rho}$ is defined by

$$
\delta_{X_{\rho}}(\varepsilon)=\inf \left\{1-\rho\left(\frac{x+y}{2}\right): x, y \in X_{\rho}, \rho(x), \rho(y) \leq 1, \rho(x-y) \geq \varepsilon\right\}, 0 \leq \varepsilon \leq 2 .
$$

In particular, if $\rho$ is convex, the $\rho$-uniform convexity of $X_{\rho}$ is defined as

$$
\varepsilon_{0}\left(X_{\rho}\right)=\sup \left\{\varepsilon \in[0,2]: \delta_{X_{\rho}}(\varepsilon)=0\right\} .
$$


Remark 1. We can easily prove that $-1 \leq \delta_{X_{\rho}}(\varepsilon) \leq 1$ and $\delta_{X_{\rho}}(0) \leq 0$.

In Banach spaces, the convexity modular $\delta_{X}(\varepsilon)$ and the smoothness modular

$$
\rho_{X}(t)=\sup \left\{\frac{\|x+y\|+\|x-y\|}{2}-1:\|x\|=1,\|y\|=1, t \geq 0\right\}
$$

are conjugate concepts. Therefore, this paper gives the definition of $\rho$-smooth modular of modular spaces by referring to the definition of smoothness modular $\rho_{X}(t)$.

Definition 5. The $\rho$-smooth modular $\rho_{X_{\rho}}(t)$ of a modular space $X_{\rho}$ is defined by

$$
\rho_{X_{\rho}}(t)=\sup \left\{\rho\left(\frac{x+y}{2}\right)+\rho\left(\frac{x-y}{2}\right)-1: x, y \in X_{\rho}, \rho(x) \leq 1, \rho(y) \leq t\right\}, t \geq 0 .
$$

Remark 2. It is true that $\min \{0, t-1\} \leq \rho_{X_{\rho}}(t) \leq 1+2 t$ and $\rho_{X_{\rho}}(t)$ is increasing of $t$.

Theorem 2. Let $X$ be a modular space, then

(i) $J\left(X_{\rho}\right)<2 \epsilon$ if and only if $\delta_{X_{\rho}}(\epsilon)>1-\epsilon$, in particular, if $\rho$ is convex, then $J\left(X_{\rho}\right)<\epsilon$ if and only if $\delta_{X_{\rho}}(\epsilon)>1-\frac{\epsilon}{2}$;

(ii) $J\left(X_{\rho}\right)=2 \sup \left\{\epsilon \in(0,2): \delta_{X_{\rho}}(\epsilon) \leq 1-\epsilon\right\}$, in particular, if $\rho$ is convex, then $J\left(X_{\rho}\right)=\sup \{\epsilon \epsilon$ $\left.(0,2): \delta_{X_{\rho}}(\epsilon)<1-\frac{\epsilon}{2}\right\}$.

Proof. (i) Note $\alpha=J\left(X_{\rho}\right)<2 \epsilon$, thus

$$
\min \left\{\rho\left(\frac{x+y}{2}\right), \rho\left(\frac{x-y}{2}\right)\right\} \leq \frac{\alpha}{2},
$$

shows that $1-\rho\left(\frac{x+y}{2}\right) \geq 1-\frac{\alpha}{2}>1-\epsilon$. Therefore $\delta_{X_{\rho}}(\epsilon)>1-\epsilon$.

Note $\beta=\delta_{X_{\rho}}(\epsilon)>1-\epsilon$, then $1-\rho\left(\frac{x+y}{2}\right) \geq \beta$ implies $\rho\left(\frac{x+y}{2}\right) \leq 1-\beta<\epsilon$. Thus

$$
\min \left\{\rho\left(\frac{x+y}{2}\right), \rho\left(\frac{x-y}{2}\right)\right\}=\rho\left(\frac{x+y}{2}\right) .
$$

Then

$$
\begin{aligned}
J\left(X_{\rho}\right)= & 2 \sup \left\{\rho\left(\frac{x+y}{2}\right): x, y \in X_{\rho}, \rho(x)=1, \rho(y) \leq 1\right\} \\
& \leq 2-2 \beta<2 \varepsilon .
\end{aligned}
$$

In particular, if $\rho$ is convex and let $\lambda=J\left(X_{\rho}\right)<\epsilon$, then $J\left(X_{\rho}\right)<\epsilon$ if and only if $\forall x, y \in$ $x_{\rho}, \rho(x), \rho(y) \leq 1$, we have

$$
\rho(x+y) \leq \lambda \text { or } \rho(x-y) \leq \lambda .
$$

According to the Definition of $\delta_{X_{\rho}}(\epsilon)$, we obtain $\rho(x+y) \geq \epsilon>\lambda$, thus $\rho(x-y) \leq \lambda$ shows that

$$
\delta_{x_{\rho}}(\epsilon) \geq 1-\frac{\alpha}{2}>1-\frac{\epsilon}{2} .
$$

(ii) Note $\epsilon_{0}=\sup \left\{\epsilon \in(0,2): \delta_{X_{\rho}}(\epsilon) \leq 1-\epsilon\right\}$.

Suppose $\epsilon_{0}<2 . \forall \epsilon \in\left(\epsilon_{0}, 2\right)$, for any $x, y \in X_{\rho}$ and $\rho(x), \rho(y) \leq 1$, we have

$$
\rho(x-y)>\epsilon \text { or } \rho(x-y) \leq \epsilon .
$$

If $\rho(x-y)>\epsilon$, then $\delta_{X_{\rho}}(\epsilon) \geq 1-\epsilon$ implies $\rho\left(\frac{x+y}{2}\right) \leq \epsilon$. Thus $J\left(X_{\rho}\right) \leq 2 \epsilon$. 
Since $\delta_{X_{\rho}}(\epsilon) \leq 1-\epsilon$, then $J\left(X_{\rho}\right) \leq 2 \epsilon_{0}$ shows that

$$
J\left(X_{\rho}\right)=2 \sup \left\{\epsilon \in(0,2): \delta_{X_{\rho}}(\epsilon) \leq 1-\epsilon\right\} .
$$

In particular, if $\rho$ is convex and let $\alpha=J\left(X_{\rho}\right) \in[1,2]$, then $\forall x, y \in X_{\rho}, \rho(x), \rho(y) \leq 1$, we have

$$
\rho(x+y) \leq \alpha \text { or } \rho(x-y) \leq \alpha
$$

What's more, $\forall \eta>0$, there exist $x^{\prime}, y^{\prime} \in X_{\rho}$ and $\rho\left(x^{\prime}\right), \rho\left(y^{\prime}\right) \leq 1$ such that

$$
\rho\left(x^{\prime}+y^{\prime}\right)>\alpha-\eta \text { and } \rho\left(x^{\prime}-y^{\prime}\right)>\alpha-\eta \text {. }
$$

Fix $\eta>0$, then $1-\rho\left(\frac{x^{\prime}+y^{\prime}}{2}\right)<1-\frac{\alpha-\eta}{2}$ implies $\delta_{X_{\rho}}(\epsilon)<1-\frac{\alpha-\eta}{2}$, therefore

$$
\sup \left\{\epsilon \in(0,2): \delta_{x_{\rho}}(\epsilon)<1-\frac{\epsilon}{2}\right\} \geq \alpha-\eta \text {. }
$$

$\forall \epsilon \in(0,2)$, if $\epsilon \leq \alpha$, thus

$$
\sup \left\{\epsilon \in(0,2): \delta_{X_{\rho}}(\epsilon)<1-\frac{\epsilon}{2}\right\} \leq \alpha
$$

If $\epsilon>\alpha$, then $\rho(x+y) \leq \alpha$ shows that $\delta_{X_{\rho}}(\epsilon) \geq 1-\frac{\alpha}{2}$. In $(0,2)$, we know

$$
\sup \left\{\epsilon \in(0,2): \delta_{X_{\rho}}(\epsilon)<1-\frac{\epsilon}{2}\right\} \leq \alpha
$$

thus $\alpha-\eta \leq \sup \left\{\epsilon \in(0,2): \delta_{X_{\rho}}(\epsilon)<1-\frac{\epsilon}{2}\right\} \leq \alpha$.

Let $\eta \rightarrow 0$, then

$$
\sup \left\{\epsilon \in(0,2): \delta_{X_{\rho}}(\epsilon)<1-\frac{\epsilon}{2}\right\}=\alpha .
$$

Theorem 3. Let $X_{\rho}$ be a modular space, then

(i) $J\left(X_{\rho}\right) \leq \rho_{X_{\rho}}(1)+1$;

(ii) $C_{N J}\left(X_{\rho}\right) \leq 2\left(\sqrt{12+\left(1+\rho_{X_{\rho}}(1)\right)^{2}}-2\right)^{2}$.

Proof. (i) We can deduce that

$$
\begin{aligned}
J\left(X_{\rho}\right) & \leq \sup \left\{\rho\left(\frac{x+y}{2}\right)+\rho\left(\frac{x-y}{2}\right): x, y \in X_{\rho}, \rho(x)=1, \rho(y) \leq 1\right\} \\
& =\rho X_{\rho}(1)+1 .
\end{aligned}
$$

(ii) We know that $a^{2}+b^{2} \leq(a+b)^{2}-4(a+b)+8$ for $0<a, b \leq 2$. Thus

$$
\rho^{2}\left(\frac{x+y}{2}\right)+\rho^{2}\left(\frac{x-y}{2}\right) \leq\left(\rho\left(\frac{x+y}{2}\right)+\rho\left(\frac{x-y}{2}\right)\right)^{2}-4\left(\rho\left(\frac{x+y}{2}\right)+\rho\left(\frac{x-y}{2}\right)\right)+8 .
$$

Since

$$
\rho\left(\frac{x+y}{2}\right)+\rho\left(\frac{x-y}{2}\right) \geq \sqrt{\rho^{2}\left(\frac{x+y}{2}\right)+\rho^{2}\left(\frac{x-y}{2}\right)}
$$


then

$$
\begin{aligned}
& \rho^{2}\left(\frac{x+y}{2}\right)+\rho^{2}\left(\frac{x-y}{2}\right)+4 \sqrt{\rho^{2}\left(\frac{x+y}{2}\right)+\rho^{2}\left(\frac{x-y}{2}\right)}-8 \\
& \leq\left(\rho\left(\frac{x+y}{2}\right)+\rho\left(\frac{x-y}{2}\right)\right)^{2} \\
& \leq\left(1+\rho x_{\rho}(1)\right)^{2} .
\end{aligned}
$$

Thus

$$
\rho^{2}\left(\frac{x+y}{2}\right)+\rho^{2}\left(\frac{x-y}{2}\right) \leq\left(\sqrt{12+\left(1+\rho_{X_{\rho}}(1)\right)^{2}}-2\right)^{2}
$$

which shows that $\frac{1}{2} C_{N J}\left(X_{\rho}\right) \leq\left(\sqrt{12+\left(1+\rho_{X_{\rho}}(1)\right)^{2}}-2\right)^{2}$.

\section{Convexity and Non-SQuareness}

Clarkson introduced uniform convexity in 1936, proved that $I_{p}(1 \leq p<\infty)$ spaces are uniformly convex Banach spaces and uniformly convex Banach spaces have Radon-Nikodym properties. Due to the geometrical intuitiveness of convexity, Poom Kumam [10] gave the definitions of $\rho_{r}$-uniformly convex, $\rho$-uniformly non-square and $\rho$-strictly convex of modular spaces in 2006.

On the basis of literature [10], this paper studies the relationships between convexity, nonsquareness and geometric constants of modular spaces.

Definition 6.[10] For $r>0$, a modular space $X_{\rho}$ is said to be $\rho_{r}$-uniformly convex if for each $\epsilon>0$, there exists $\delta>0$ such that for any $x, y \in X_{\rho}$, the conditions $\rho(x) \leq r, \rho(y) \leq r$ and $\rho(x-y) \geq r \epsilon$ imply that $\rho\left(\frac{x+y}{2}\right) \leq(1-\delta) r$.

Definition 7.[10] The modular space $X_{\rho}$ is said to be $\rho$-uniformly non-square if there exists $\delta \in(0,1)$ such that for any $x, y \in X_{\rho}$ with $\rho(x)=1$ and $\rho(y) \leq 1, \rho\left(\frac{x+y}{2}\right) \leq 1-\delta$ or $\rho\left(\frac{x-y}{2}\right) \leq 1-\delta$.

Definition 8.[10] The modular space $X_{\rho}$ is said to be $\rho$-strictly convex if for any $x, y \in X_{\rho}$, the conditions $\rho(x) \leq 1, \rho(y) \leq 1$ and $x \neq y$ imply that $\rho\left(\frac{x+y}{2}\right)<1$.

Theorem 4. Let $X_{\rho}$ be a modular space, then the following conditions are equivalent.

(i) $J\left(X_{\rho}\right)<2$;

(ii) $\epsilon_{0}\left(X_{\rho}\right)<2$ for all $0<\epsilon \leq 2$;

(ii) $X_{\rho}$ is $\rho$-uniformly non-square.

Proof. Suppose $J\left(X_{\rho}\right)<2$. There exists $\epsilon>0$, for any $x, y \in X_{\rho}$ with $\rho(x)=1$ and $\rho(y) \leq 1$, such that

$$
\rho\left(\frac{x+y}{2}\right) \leq \frac{J\left(X_{\rho}\right)}{2}-\epsilon<1-\epsilon \text { or } \rho\left(\frac{x-y}{2}\right) \leq \frac{J\left(X_{\rho}\right)}{2}-\epsilon<1-\epsilon,
$$

implies $X_{\rho}$ is $\rho$-uniformly non-square.

Suppose $X_{\rho}$ is $\rho$-uniformly non-square, then we can prove $J\left(X_{\rho}\right)<2$ by the same way. Thus (i) and (iii) are equivalent.

Next, we know that $\epsilon_{0}\left(X_{\rho}\right)<2$ if and only if $\delta_{X_{\rho}}(2)>0$. Let $\alpha=\delta_{X_{\rho}}(2)$, then $\forall x, y \in$ $X_{\rho}$ and $\rho(x)=1, \rho(y) \leq 1$, we can get $\rho\left(\frac{x \pm y}{2}\right) \leq 1-\alpha$, thus $X_{\rho}$ is $\rho$-uniformly non-square. Thus 
(ii) and (iii) are equivalent.

Remark 3. In fact, this theorem is a generalization of Theorem 3.8 in [11].

Theorem 5. Let $X$ be a modular space, then

(i) $X$ is $\rho_{1}$-uniformly convex if and only if $\delta_{X_{\rho}}(\epsilon)>0$ for $0<\epsilon \leq 2$;

(ii) If $\delta_{X_{\rho}}(2)=1$, then $X_{\rho}$ is $\rho$-strictly convex.

Proof. (i) Denote $\delta_{\varepsilon}=\delta_{X_{\rho}}(\epsilon)$. Then $\delta_{X_{\rho}}(\epsilon)>0$ if and only if $\forall x, y \in X_{\rho}, \rho(x), \rho(y) \leq 1$ and $\rho(x-y) \geq \epsilon$, we have $\rho\left(\frac{x+y}{2}\right) \leq 1-\delta_{\epsilon}$. Thus $X_{\rho}$ is $\rho_{1}$-uniformly convex.

(ii) Since $\delta_{X_{\rho}}(2)=1$, then $\forall x, y \in X_{\rho}, \rho(x), \rho(y) \leq 1$ and $\rho(x-y) \geq 2$, we have

$$
\rho\left(\frac{x+y}{2}\right)=0<1
$$

implies $X_{\rho}$ is $\rho$-strictly convex.

\section{Midpoint Convexity}

In the following section, we discuss a special type of modular and study its properties in terms of geometric constants.

Definition 9.[6] Let $(X,\|\cdot\|)$ be a normed space and $X_{\rho}$ be a modular space. Then $\rho$ is said to be strongly midpoint convex with non-negtive constant $C$ if

$$
\rho\left(\frac{x+y}{2}\right) \leq \frac{\rho(x)+\rho(y)}{2}-\frac{C}{4}\|x-y\|^{2} .
$$

Theorem 6. Let $(X,\|\cdot\|)$ be a normed space and $X_{\rho}$ be a modular space. If there exists $C \geq 0$ such that

$$
C\|x\|^{2} \leq \frac{1}{2} \rho(x) \text { for all } x \in B_{x_{\rho}}
$$

and $\rho$ is strongly midpoint convex with constant $C_{\text {, then }} C_{N J}\left(X_{\rho}\right) \leq 3$.

Proof. Since $\rho\left(\frac{x+y}{2}\right) \leq \frac{\rho(x)+\rho(y)}{2}-\frac{C}{4}\|x-y\|^{2}$ and $\rho\left(\frac{x-y}{2}\right) \leq \frac{\rho(x)+\rho(-y)}{2}-\frac{C}{4}\|x+y\|^{2}$, then

$$
\rho^{2}\left(\frac{x+y}{2}\right) \leq \frac{1}{4}(\rho(x)+\rho(y))^{2}-\frac{C}{4}\|x-y\|^{2}(\rho(x)+\rho(y))+\frac{C^{2}}{16}\|x-y\|^{4}
$$

and

$$
\rho^{2}\left(\frac{x-y}{2}\right) \leq \frac{1}{4}(\rho(x)+\rho(y))^{2}-\frac{C}{4}\|x+y\|^{2}(\rho(x)+\rho(y))+\frac{C^{2}}{16}\|x+y\|^{4} .
$$

Therefore, for $x \in S_{X_{\rho}}$ and $y \in B_{X_{\rho}}$, we have

$$
\begin{aligned}
\rho^{2}\left(\frac{x+y}{2}\right)+\rho^{2}\left(\frac{x-y}{2}\right) & \leq \frac{1}{2}(1+\rho(y))^{2}-\frac{C}{4}(1+\rho(y))\left(\|x+y\|^{2}+\|x-y\|^{2}\right)+ \\
& \frac{C^{2}}{16}\left(\|x+y\|^{4}+\|x-y\|^{4}\right)
\end{aligned}
$$

Next, we only need to prove

$$
\rho(y)+\frac{C^{2}}{16}\left(\|x+y\|^{4}+\|x-y\|^{4}\right)-\frac{C}{4}\left(\|x+y\|^{2}+\|x-y\|^{2}\right)(1+\rho(y))-1-\rho^{2}(y) \leq 0 .
$$


Let $t=\|x+y\|^{2}+\|x-y\|^{2}, s=\|x+y\|\|x-y\|$ and $I_{1}=\rho(y)+\frac{C^{2}}{16}\left(t^{2}-2 s^{2}\right)-\frac{C}{4} t(1+$ $\rho(y))-1-\rho^{2}(y)$, then

$$
I_{1} \leq \frac{t^{2}-2 s^{2}}{16} C^{2}-\frac{t}{4} C=\frac{C\left(t^{2}-2 s^{2}\right)}{16}\left(C-\frac{4 t}{t^{2}-2 s^{2}}\right) .
$$

Since $C\|x\|^{2} \leq \frac{1}{2} \rho(x)$, then $C\left\|\frac{x+y}{2}\right\|^{2} \leq \frac{1}{2} \rho\left(\frac{x+y}{2}\right) \leq 1$ and $C\left\|\frac{x-y}{2}\right\|^{2} \leq \frac{1}{2} \rho\left(\frac{x-y}{2}\right) \leq 1$.

Therefore

$$
\frac{4 t}{t^{2}-2 s^{2}}=\frac{\left\|\frac{x+y}{2}\right\|^{2}+\left\|\frac{x-y}{2}\right\|^{2}}{\left\|\frac{x+y}{2}\right\|^{4}+\left\|\frac{x-y}{2}\right\|^{4}} \geq \frac{1}{\left\|\frac{x+y}{2}\right\|^{2}+\left\|\frac{x-y}{2}\right\|^{2}} \geq C
$$

then $I_{1} \leq 0$.

Example 3. If $\rho$ is convex, then $C=0$ which satisfies the condition of Theorem 6 , and $C_{N J}\left(X_{\rho}\right) \leq$ $2<3$.

Theorem 7. Let $(X,\|\cdot\|)$ be a normed space and $X_{\rho}$ be a modular space. If there exist $C, \lambda, \mu, \gamma>0$ such that

$$
2 \mu \gamma \leq 1 \leq \frac{1}{2 \lambda}+\frac{\sqrt{6}}{8 \mu} \text { and } \mu \rho(x) \leq C\|x\|^{2} \leq \lambda \rho(x) \text { for all } x \in X_{\rho} .
$$

What'more, $\rho$ is strongly midpoint convex with constant $C$, then $C_{N J}\left(X_{\rho}\right) \leq 2$.

Proof. By following the ideas in Theorem 6, we can get

$$
\begin{aligned}
\frac{\rho^{2}\left(\frac{x+y}{2}\right)+\rho^{2}\left(\frac{x-y}{2}\right)}{1+\rho^{2}(y)} & \leq \frac{1}{2}+\frac{1}{1+\rho^{2}(y)}\left\{\rho(y)-\frac{C}{4}(1+\rho(y))\left(\mid x+y\left\|^{2}+\right\| x-y \|^{2}\right)\right\} \\
& +\frac{1}{1+\rho^{2}(y)}\left\{\frac{C^{2}}{16}\left(\|x+y\|^{4}+\|x-y\|^{4}\right)\right\} .
\end{aligned}
$$

Let

$$
t=\|x+y\|^{2}+\|x-y\|^{2}, s=\|x+y\|\|x-y\|
$$

and

$$
I_{2}=\frac{t^{2}-2 s^{2}}{16} C^{2}-\frac{(1+\rho(y)) t}{4} C-\frac{1}{2}(1-\rho(y))^{2},
$$

thus we only need to prove $I_{2} \leq 0$. Since $\rho(y) \leq 1$, then

$$
(1+\rho(y)) t-\sqrt{(1+\rho(y))^{2} t^{2}+2\left(t^{2}-2 s^{2}\right)(1-\rho(y))^{2}} \leq t
$$

and $(1+\rho(y)) t+\sqrt{(1+\rho(y))^{2} t^{2}+2\left(t^{2}-2 s^{2}\right)(1-\rho(y))^{2}} \geq t+\sqrt{3 t^{2}-4 s^{2}}$.

Thus

$$
\frac{t}{t^{2}-2 s^{2}}=\frac{1}{4} \cdot \frac{\left\|\frac{x+y}{2}\right\|^{2}+\left\|\frac{x-y}{2}\right\|^{2}}{\left\|\frac{x+y}{2}\right\|^{4}+\left\|\frac{x-y}{2}\right\|^{4}}
$$

and

$$
\begin{aligned}
& \frac{t+\sqrt{3 t^{2}-4 s^{2}}}{t^{2}-2 s^{2}} \\
& =\frac{1}{4} \cdot \frac{\|x+y\|^{2}+\left\|\frac{x-y}{2}\right\|^{2}+\sqrt{3\left\|\frac{x+y}{2}\right\|^{4}+3\left\|\frac{x-y}{2}\right\|^{4}+2\left\|\frac{x+y}{2}\right\|^{2}\left\|\frac{x-y}{2}\right\|^{2}}}{\left\|\frac{x+y}{2}\right\|^{4}+\left\|\frac{x-y}{2}\right\|^{4}},
\end{aligned}
$$


then

$$
\begin{gathered}
\frac{t+\sqrt{3 t^{2}-4 s^{2}}}{t^{2}-2 s^{2}} \geq \frac{1}{4\left\|\frac{x+y}{2}\right\|^{2}+4\left\|\frac{x-y}{2}\right\|^{2}}+\frac{\sqrt{3}}{4 \sqrt{\left\|\frac{x+y}{2}\right\|^{4}+\left\|\frac{x-y}{2}\right\|^{4}}} \\
\geq \frac{C}{\lambda\left(\rho\left(\frac{x+y}{2}\right)+\rho\left(\frac{x-y}{2}\right)\right)}+\frac{\sqrt{3} C}{4 \mu \sqrt{\rho^{2}\left(\frac{x+y}{2}\right)+\rho^{2}\left(\frac{x-y}{2}\right)}} \\
\geq \frac{C}{4 \lambda}+\frac{\sqrt{3} C}{8 \sqrt{2} \mu} \geq \frac{C}{2},
\end{gathered}
$$

and

$$
\frac{t}{t^{2}-2 s^{2}} \leq \frac{1}{2} \cdot \frac{1}{\left\|\frac{x+y}{2}\right\|^{2}+\left\|\frac{x-y}{2}\right\|^{2}} \leq \frac{C}{4 \mu\left(\rho\left(\frac{x+y}{2}\right)+\rho\left(\frac{x-y}{2}\right)\right)} \leq \frac{C}{4 \mu \gamma} \leq \frac{C}{2} .
$$

Therefore

$$
\begin{aligned}
& \frac{(1+\rho(y)) t-\sqrt{(1+\rho(y))^{2} t^{2}+2\left(t^{2}-2 s^{2}\right)(1-\rho(y))^{2}}}{t^{2}-2 s^{2}} \\
& \leq \frac{C}{2} \leq \\
& \frac{(1+\rho(y)) t+\sqrt{(1+\rho(y))^{2} t^{2}+2\left(t^{2}-2 s^{2}\right)(1-\rho(y))^{2}}}{t^{2}-2 s^{2}}
\end{aligned}
$$

Thus

$$
\begin{aligned}
I_{2}= & \frac{t^{2}-2 s^{2}}{4}\left(\frac{C}{2}-\frac{(1+\rho(y)) t+\sqrt{(1+\rho(y))^{2} t^{2}+2\left(t^{2}-2 s^{2}\right)(1-\rho(y))^{2}}}{t^{2}-2 s^{2}}\right) \\
& \left(\frac{C}{2}-\frac{(1+\rho(y)) t-\sqrt{(1+\rho(y))^{2} t^{2}+2\left(t^{2}-2 s^{2}\right)(1-\rho(y))^{2}}}{t^{2}-2 s^{2}}\right)
\end{aligned}
$$

$\leq 0$

Example 4. Consider $\rho(x)=4 C\|x\|^{2}$ and let $\lambda=\mu=\frac{1}{4}$, then $\mu^{2} \rho(x) \leq C\|x\|^{2} \leq \lambda \rho(x)$ and $\mu^{2}=\frac{\lambda}{4}$. What'more,

$$
\begin{aligned}
C_{N J}\left(X_{\rho}\right) & =2 C \sup \left\{\frac{\|x+y\|^{4}+\|x-y\|^{4}}{\|x+y\|^{2}+\|x-y\|^{2}}: x, y \in X_{\rho}, \rho(x)=1, \rho(y) \leq 1\right\} \\
& \leq 2 C \sup \left\{\|x+y\|^{2}+\|x-y\|^{2}: x, y \in X_{\rho}, \rho(x)=1, \rho(y) \leq 1\right\} \\
& \leq 4 C \sup \left\{\|x\|^{2}+\|y\|^{2}: x, y \in X_{\rho}, \rho(x)=1, \rho(y) \leq 1\right\} \\
& =\sup \left\{\rho(x)+\rho(y): x, y \in X_{\rho}, \rho(x)=1, \rho(y) \leq 1\right\}=2 .
\end{aligned}
$$

Theorem 8. Let $(X,\|\cdot\|)$ be a normed space, $X_{\rho}$ be a modular space and $\alpha_{0} \in(0,2 \sqrt{2}]$. If there exists $C>0$ such that

$$
C \geq \frac{4 \alpha_{0}}{\sqrt{\left\|x_{0}+y_{0}\right\|^{4}+\left\|x_{0}-y_{0}\right\|^{4}}} \text { for some } x_{0} \in S_{x_{\rho}}, y_{0} \in B_{X_{\rho}}
$$

and $\rho$ is strongly midpoint convex with positive constant $C_{\text {, then }} C_{N J}\left(X_{\rho}\right) \geq \alpha_{0}^{2}$. In particular, if $\alpha_{0}=2 \sqrt{2}$, then $C_{N J}\left(X_{\rho}\right)=8$.

Proof. Since $2 \rho(x) \geq C\|x\|^{2}$, then $\rho^{2}\left(\frac{x \pm y}{2}\right) \geq \frac{C}{2}\left\|\frac{x \pm y}{2}\right\|^{2}$. Therefore

$$
\frac{\rho^{2}\left(\frac{x+y}{2}\right)+\rho^{2}\left(\frac{x-y}{2}\right)}{1+\rho^{2}(y)} \geq \frac{C^{2}}{16} \frac{\left(\|x+y\|^{4}+\|x-y\|^{4}\right)}{1+\rho^{2}(y)},
$$


shows that $\frac{\rho^{2}\left(\frac{x+y}{2}\right)+\rho^{2}\left(\frac{x-y}{2}\right)}{1+\rho^{2}(y)} \geq \frac{\alpha_{0}^{2}}{1+\rho^{2}(y)}$, then $C_{N J}\left(X_{\rho}\right) \geq \alpha_{0}^{2}$. If $\alpha_{0}=2 \sqrt{2}$, then $C_{N}\left(X_{\rho}\right) \geq 8$ implies $C_{N J}\left(X_{\rho}\right)=8$.

\section{Data Availability}

No data were used to support this study.

\section{Conflicts of InTERESt}

The author(s) declare(s) that there is no conflict of interest regarding the publication of this paper.

\section{Funding Statement}

This work was supported by the National Natural Science Foundation of P. R. China (Nos. 11971493 and 12071491).

\section{REFERENCES}

[1] J. Lindenstrauss, On the modulus of smoothness and divergent series in Banach spaces, Michigan Math. J. 10 (1963). https://doi.org/10.1307/mmj/1028998906.

[2] J.A. Clarkson, Uniformly convex spaces, Trans. Amer. Math. Soc. 40 (1936) 396-396. https://doi.org/10.1090/ S0002-9947-1936-1501880-4.

[3] J.A. Clarkson, The Von Neumann-Jordan Constant for the Lebesgue Spaces, The Annals of Mathematics. 38 (1937) 114. https://doi.org/10.2307/1968512.

[4] R.C. James, Uniformly Non-Square Banach Spaces, The Annals of Mathematics. 80 (1964) 542. https: //doi . org/ $10.2307 / 1970663$.

[5] E. L. Fuster, Moduli and constants-What a show! (2006). https://www. uv. es/1lorens/Documento.pdf.

[6] J. Musielak, W. Orlicz, On modular spaces, Studia Math. 18 (1959) 49-65. https://doi.org/10.4064/ sm-18-1-49-65.

[7] H. Nakano, Modulared semi-ordered linear spaces, Tokyo, Maruzen Co.Ltd. (1950).

[8] M.A. Khamsi, W.M. Kozlowski, Fixed Point Theory in Modular Function Spaces, Springer International Publishing, Cham, 2015. https://doi.org/10.1007/978-3-319-14051-3.

[9] J. Musielak, Orlicz spaces and Modular spaces.Lecture Note in math, Springer-Verlag, Berlin, Heidelberg, New York, 1034. (1983).

[10] P. Kumam, Fixed Point Property in Modular Spaces.Master Thesis, Chiang Mai University Thailand, (2002).

[11] P. Kumam, On Nonsquare and Von Neumann Constants of Modular Spaces, Southeast Asian Bull. Math. 30 (2006), 69-77.

[12] P. Kumam, Some Geometric Properties and Fixed Point Theorem in Modular Spaces, International Conference on Fixed Point Theory and Applications, 173-188. (2003).

[13] C. Yang, A note on Jordan-von Neumann constant and James constant, J. Math. Anal. Appl. 357 (2009) 98-102. https://doi.org/10.1016/j.jmaa.2009.04.002.

[14] C. Yang, An inequality between the James type constant and the modulus of smoothness, Journal of Mathematical Analysis and Applications. 398 (2013) 622-629. https://doi.org/10.1016/j.jmaa. 2012.07.063. 
[15] G. Nordlander, The modulus of convexity in normed linear spaces, Ark. Mat. 4 (1960) 15-17. https://doi.org/ $10.1007 / \mathrm{BF} 02591317$.

[16] K. Nikodem, Z. Pales, Characterizations of inner product spaces by strongly convex functions, Banach J. Math. Anal. 5 (2011) 83-87. https://doi.org/10.15352/bjma/1313362982. 